\title{
Corticosteroid-induced psychosis and formication in a male with eczema using topical triamcinolone
}

\author{
Mihir U*, Modupe J and Venkateshwara M \\ BronxCare Health System, Icahn School of Medicine at Mount Sinai, Bronx, New York, USA
}

\begin{abstract}
Systemic corticosteroids used to treat various autoimmune conditions have long been associated with adverse psychiatric effects. Symptoms such as euphoria, insomnia, mood swings, personality changes, severe depression, and psychosis, referred to as corticosteroid-induced psychosis, have been estimated to develop in $5 \%$ to $18 \%$ of patients treated with corticosteroids. Triamcinolone is a topical corticosteroid that is the standard of care for treating eczema, a form of atopic dermatitis that usually develops in early childhood. It is not typically implicated in the exacerbation of psychiatric symptoms, but it is theorized that long-term use of triamcinolone by an already-immunocompromised individual may render such an effect. Here, we outline the case of a 26-year-old African American male who came to the emergency room in acute distress and formication (tactile hallucinations), feeling that mites were crawling underneath his skin. He had deep scratch marks on every aspect of his body and had used a razor to make cuts on all of his limbs, hoping the mites would "come out." Upon interview, it was learned he had been overdosing on topical triamcinolone for five years in an attempt to control exacerbation of eczema on various parts of his body. The patient was admitted to the inpatient psychiatric unit, withdrawn from triamcinolone, and started on antipsychotic medication. The patient returned to psychiatric baseline over the next few days. In studying this case, we outline how the potency of corticosteroids combined with their misuse can lead to psychosis.
\end{abstract}

\section{Introduction}

Systemic corticosteroids used to treat various autoimmune conditions have long been associated with adverse psychiatric effects. Symptoms such as euphoria, insomnia, mood swings, personality changes, severe depression, and psychosis, referred to as corticosteroidinduced psychosis, have been estimated to develop in $5 \%$ to $18 \%$ of patients treated with corticosteroids. Mania and hypomania are the most commonly reported symptoms (35\%), followed by depressive symptoms (28\%) and psychotic reactions (24\%) [1]. Psychiatric symptoms typically arise within a week of starting corticosteroid therapy, though it is entirely possible for symptoms to commence at any time, including after the corticosteroid is discontinued [2]. They may last for a matter of days or persist for several weeks.

Triamcinolone is an intermediate-acting glucocorticoid given orally, by injection, inhalation, or as a topical ointment or cream. The topical form of this corticosteroid is the standard of care for treating eczema, a form of atopic dermatitis that usually develops in early childhood. It is relatively less potent compared to other corticosteroids, and topical application of the medication is less precarious than oral, intramuscular, or intravenous dosing. For these reasons, triamcinolone is not typically implicated in the manifestation or exacerbation of psychiatric symptoms. However, the possibility still exists, and it has been theorized that long-term use of triamcinolone by an alreadyimmunocompromised individual may render such an effect.

Here, we outline the case of a 26-year-old African American male with HIV and chronic eczema who came to the emergency room in acute distress and endorsing formication (tactile hallucinations). Upon interview, it was learned he had been overdosing on topical triamcinolone for five years in an attempt to control exacerbation of eczema on various parts of his body. In studying this case, we outline how the potency of corticosteroids combined with the immunocompromised state caused by HIV can make an individual prone to psychosis.

\section{Case report}

D.W. is a 26-year-old African American male residing in the inner city of a major metropolitan city on in the East Coast of the United States. He has a history of HIV and substance-induced mood disorder, in addition to chronic eczema on his face, neck, and various parts of his torso and limbs.

On this occasion, D.W. presented to the emergency department at 2:00 AM, brought in by ambulance that he activated. He was anxious and irritable upon arrival and was bleeding from multiple superficial cuts to his left arm, abdomen, and right leg, all of which were selfinflicted.

However, he was adamant he had no intention of hurting himself, saying, "These cuts won't kill me, the bugs under my skin will." Restless, he had difficulty sitting still and spoke with labile affect. He further complained that he hadn't slept much for days, explaining he was too anxious to fall asleep. He agonized, "Bugs are crawling all over my body! Every part. Even my private area. They're biting me, so I took a razor and tried to get them off." He insisted that he had contracted scabies, based on a Google search of the most likely species of mites implicated in skin infestation. He had obtained a tube of Permethrin cream though means he wouldn't divulge but finished the entire tube in a day. His motivation for coming to the hospital was to get a prescription for more Permethrin.

${ }^{*}$ Correspondence to: Mihir Upadhyaya, MD, BronxCare Health System, Icahn School of Medicine at Mount Sinai, Bronx, New York, USA, E-mail: mupadhya@bronxleb.org

Received: October 15, 2018; Accepted: October 24, 2018; Published: October 26,2018 
D.W. was admitted to the psychiatric inpatient unit, where it was learned he hadn't taken his anti-retroviral medication to treat his HIV for several months and was using triamcinolone cream to treat his eczema. He was re-started on both, in addition to an atypical antipsychotic medication to address his psychosis. In the days after admission, he continued to be agitated and irritable, often pacing the halls of the inpatient unit and getting into verbal altercations with staff and peers. He also remained convinced that bugs were still crawling under his skin, and he began to surreptitiously use plastic eating utensils to cut his skin to "let the bugs out." This behaviour required the patient to be placed on constant observation.

Over the first few days of hospitalization, the dosing of triamcinolone cream was a constant point of contention, as D.W. insisted that application twice a day was simply not enough, and he wasn't getting nearly the amount of cream he wanted. After days of frustration, D.W. finally admitted he's been using the steroid cream for years as a "beauty product," and believed it helps "replenish" his skin. "I lather it on like sunscreen, multiple times a day," he acknowledged. He has gone to more than six doctors in the past six years to ask for a prescription for triamcinolone and would seek out a new doctor if one refuses to prescribe it. He often went to emergency rooms to get a prescription if he wasn't able to obtain an office appointment in a timely manner. In recent months, D.W. has also learned to purchase the medication from online pharmacies in other countries where no prescription is needed but was reluctant to continue buying it from abroad because it's far more expensive.

A medical consult was called to determine the appropriateness of further use of triamcinolone, and the medication was immediately withdrawn. There were several days of pleading and protests from D.W. after the medication was stopped. He required emergent medication on an almost-daily basis for nearly a week, as his frustration with not receiving triamcinolone agitated him to the point of physical aggression toward the staff, including times when he spat on nurses. He would also engage in temper tantrums where he would throw himself on the ground, bang his fists against the floor, and sob loudly and uncontrollably. He feared the eczema would return. However, he also admitted the feeling of bugs crawling under his skin had gradually resolved. In addition to discontinuing the corticosteroid, the patient was started on Risperidone, an atypical antipsychotic medication. This medication was started at the time of hospitalization and had been titrated up to 3 milligrams twice a day at the time of discharge.

D.W. spent 11 days in the inpatient psychiatric unit, and at the end of his hospitalization, he was better able to sit still and engage in calm, logical conversation. Once the mechanism of action of triamcinolone was explained to him, and how it was a known cause of psychotic behaviour, he agreed to stop using it. He was discharged from the psychiatric unit with instructions to continue his antipsychotic medication and follow up with an outpatient psychiatric, in addition to following up with a dermatologist to devise a treatment plan for his eczema that avoided overuse of corticosteroids.

\section{Discussion}

The patient's obsession with treating his eczema is a well-known phenomenon in the field of dermatology, and the term Topical Steroid Addiction (TSA) was coined in 1973, defined as "the situation where skin develops more severe or diverse skin manifestations after the withdrawal from topical corticosteroids than at preapplication." TSA has no psychiatric implication, per se, as the "addiction" applies to the skin itself, and not the individual afflicted with the dermatitis [3]. While research into the condition is scarce, it is postulated that higher potency of corticosteroids and longer periods of application are more likely to induce TSA. D.W. admitted to using triamcinolone for multiple years to treat eczema and went to great lengths to secure an abundance of the medication. Given his history of alternating between doctors willing to prescribe the corticosteroid, and then resorting to buying it online without a prescription from offshore pharmacies, there is no way to quantify the amount of medication.

D.W. used, his admission that he would "lather" it on multiple times a day like sunscreen constitutes a vast overdose. It is reasonable to believe the eczematous parts of D.W.s skin was "addicted" to triamcinolone, and his skin condition would quickly exacerbate if the medication were withdrawn. The long-term cure for TSA is unknown, but it obviously must start with the discontinuation of the corticosteroid, with the reasonable assumption that gradual tapering of the medication would be less likely to cause rebound exacerbation of atopic dermatitis than immediate cessation.

There is a well-known connection between corticosteroid medication and suppression of the hypothalamo-pituitary-adrenal (HPA) axis, a neuroendocrine system that regulates the body's reaction to stress and controls many body processes, including mood regulation. The HPA axis is activated by stressors, initiating an immune response in which high levels of glucocorticoids are released, suppressing immune response by inhibiting the expression of proinflammatory cytokines (e.g. IL-1, TNF alpha, and IFN gamma) and increasing the levels of anti-inflammatory cytokines (e.g. IL-4, IL-10, and IL-13) in immune cells, such as monocytes and neutrophils. For example, syndromes involving excess or inadequate cortisol production can have psychiatric manifestations. Cushing's syndrome is associated with anxiety, euphoria, depression, and psychosis, whereas Addison's disease can produce fatigue, low energy, decreased appetite, and symptoms consistent with neurovegetative symptoms of depression. HPA suppression with use of topical corticosteroids is a rare occurrence, and often overlooked [4]. D.W.'s presentation blurred the line between systemic and topical, as he applied excessive amounts of triamcinolone even after formication had begun, and he deliberately cut his skin with a razor in a psychotically driven attempt to "let the bugs out."

While the level of HPA suppression can't be quantified, it is conceivable that use of the medication may have caused suppression in both the initial topical over-application, and further exacerbation once it entered the circulation through the self-inflicted cuts.

While research into the correlation is still in its early stages, there is published evidence to show that prolonged use of systemic corticosteroid medication (more than six months) is correlated with a decrease in hippocampal size, which is then implicated in symptomatology that includes memory loss, hallucinations, depression, and vulnerability to psychological trauma. It is further believed that discontinuation of the offending medication would reverse the damage to the hippocampus, with the expectation that symptoms would also resolve [5]. While neuroimaging studies were not obtained during D.W.s hospitalization, it is conceivable that over-use of topical corticosteroids for years at a time might make him similarly susceptible to neuroanatomical damage, which might then account for the psychotic features he exhibited when he first arrived in the hospital emergency department.

The definitive solution for corticosteroid-induced psychosis is removal of the offending agent, either with a gradual tapering of the medication, or as in D.W.s case, complete and immediate discontinuation. Assuming the psychosis was entirely attributable 
to use of corticosteroids, psychotic symptoms should resolve in a matter of days, though the timeframe for return to baseline is partially dependent on the length of time and dose at which the medication was used. Depending on the clinical presentation, an antipsychotic or mood stabilizer may be added to assist in achieving baseline status. D.W. was started on Risperidone because there is published literature suggesting its efficacy in patient naïve to psychotropic medication, including adolescents. The patient should follow up with a psychiatrist as outpatient, and after a period in which psychotic symptoms have resolved, the antipsychotic medication may be tapered and withdrawn [6-7].

\section{Conclusion}

The case of D.W. brings two salient issues to light. First, the need to properly assess a patient's medical history and personal motivations in developing a diagnosis and determining a course of treatment. Based on the patient's self-injurious behaviour and psychotic features, it was assumed he was engaging in suicidality after use of recreational substances. It wasn't until days later when a thorough conversation revealed the years-long overuse of topical triamcinolone that a diagnosis of corticosteroid-induced psychosis was established. Second, the importance of educating patients on the medications they're prescribed, and the harm that may befall them with incorrect use. D.W. underestimated the potency of triamcinolone and used it as a beauty product. It was only after years of engaging in nefarious activity to obtain the medication and finally ending up in the hospital for psychiatric exacerbation that a doctor engaged him in a conversation about his use of triamcinolone that he understood the consequences of his behaviour. D.W. was receptive to working with a dermatologist to identify alternative treatments for his eczema.

Proper communication between health care providers and patients about correct use of medication can prevent misuse and help patients identify side effects and seek appropriate help to avoid exacerbation of adverse events.

\section{References}

1. Cerullo MA (2006) Corticosteroid-induced mania: Prepare for the unpredictable Current Psychiatry

2. $5: 43-50$

3. Gagliardi JP, Muzyk AJ, Holt S (2010) When Steroids Cause Psychosis. The Rheumatologist.

4. Fukaya M (2014) Topical steroid addiction in atopic dermatitis. Drug, Healthcare and Patient Safety

5. 6: 131-138. [Crossref]

6. Brown WA, Johnston R, Mayfield D (1979) The 24-hour dexamethasone suppression test in a clinical setting: Relationship to diagnosis, symptoms, and response to treatment. American Journal of Psychiatry 136: 543-547. [Crossref]

7. Brown ES, Woolston DJ, Frol A (2004) Hippocampal volume, spectroscopy, cognition, and mood in patients receiving corticosteroid therapy. Biological Psychiatry 55: 538545. [Crossref]

8. Bag O (2012) Steroid-induced psychosis in a child: treatment with risperidone. General Hospital Psychiatry 34: 103.e5-103.e6. [Crossref]

9. Muzyk AJ (2010) Corticosteroid psychosis: Stop therapy or add psychotropics? Current Psychiatry 9: 61-68.

Copyright: (C2018 Mihir U. This is an open-access article distributed under the terms of the Creative Commons Attribution License, which permits unrestricted use, distribution, and reproduction in any medium, provided the original author and source are credited. 